\title{
Probabilistic Web Data Management
}

\author{
Lei Chen • Ihab Ilyas $\cdot$ Christopher Re $\cdot$ Xiaofang Zhou
}

Published online: 23 February 2013

(C) Springer Science+Business Media New York 2013

With the development of Web 2.0 technology, enormous data are generated every day. Among these data, there exist quite a lot uncertainty due to careless data entry, incomplete information, and inconsistency among different data description. Although significant effort has been paid to find effective and efficient solutions for managing and mining general uncertain data, little attention is paid to manage uncertain data on the Web. This special issue is proposed to attract research attempts on handling uncertainty of the Web data. This special issue has attracted 12 submissions, after two rounds of very careful reviews by domain experts, we accepted three excellent papers. These three papers present new ideas to address issues on Probabilistic Web Data Management.

The first paper, "an efficient approach to suggesting topically related Web queries using hidden topic model”, authored by Lin Li, Guandong Xu, Zhenglu Yang, Peter Dolog, Yanchun Zhang and Masaru Kitsuregawa, is about handling the uncertainty of queries on the Web [3]. Often, it is hard for users to formulate an appropriate query. The best strategy is to suggest Web queries that related to the initial inquiry. However, there are quite some uncertainty existed on measuring the similarity between queries. In this paper, the authors proposed a solution based on the hidden topics. Specifically, they first built a hidden topic model, and then the trained model was used to infer the topic distribution of the newly input query. The query similarity was measured through the topic distributions, based on which, a suggestion list for the candidate queries was computed. The experimental study verified the effectiveness of the proposed approach in measuring the similarity between queries.

The second paper, "Efficient processing of top-k twig queries over probabilistic XML data", authored by Bo Ning, Chengfei Liu, and Jeffrey Xu Yu, addressed the twig query processing over probabilistic XML data [1]. Compared to certain XML data, each twig answer in probabilistic XML data is associated with a probabilistic value. Since the solutions for certain XML data do not consider uncertainty, they cannot be used for queries over probabilistic XML data. In this paper, the authors proposed a new encoding scheme, called

L. Chen $(\bowtie) \cdot$ I. Ilyas $\cdot$ C. Re $\cdot$ X. Zhou

Department of Computer Science and Engineering, Hong Kong University of Science and Technology,

Clear Water Bay, Kowloon, Hong Kong

e-mail: leichen@cse.ust.hk 
PEDewey, for probabilistic XML data and two search algorithms to find top-k probabilities of twig query answers based on document order and element order, respectively. The authors well demonstrated the solutions by extensive experiments on real and synthetic data.

The third paper, "“Querying and ranking incomplete twigs in probabilistic XML", authored by Jian Liu, Z. M. Ma, and Li Yan, is also about queries over probabilistic XML data [2]. However, the authors addressed a very interesting problem, how to return incomplete answers from probabilistic XML data efficiently. Compared to complete answers, incomplete ones may be more useful due to the high confidence and flexibly to be extended to the answers of other queries. Clearly, the search space of finding incomplete answer increased significantly, thus, the authors proposed an extended Dewey scheme for probabilistic XML data and effective search algorithm for ranking the incomplete answers. The experimental results showed a significant efficiency improvement compared to a straightforward probability-based ranking approach.

Finally, we would like to thank the authors for their high-quality contributions to this special section and the referees for their generous support and valuable suggestions.

We also like to thank Prof. Yanchun Zhang, the Editor-in-Chief of WWJ, for giving us the opportunity to publish this special issue.

\section{References}

1. Bo Ning, Chengfei Liu, and Jeffrey Xu Yu.: "Efficient processing of top-k twig queries over probabilistic XML data", in World Wide Web Journal, doi: 10.1007/s11280-011-0144-2

2. Jian Liu, Z. M. Ma, and Li Yan.: "Querying and ranking incomplete twigs in probabilistic XML", in World Wide Web Journal, doi: 10.1007/s11280-011-0149-x

3. Lin Li, Guandong Xu, Zhenglu Yang, Peter Dolog, Yanchun Zhang and Masaru Kitsuregawa.: "An efficient approach to suggesting topically related web queries using hidden topic model", in World Wide Web Journal, doi: 10.1007/s11280-011-0151-3 